\title{
Article \\ Effect of Pd Ions on the Generation of Ag and Au Heterogeneous Nanoparticles Using Laser Ablation in Liquid
}

\author{
Sangwoo Yoon ${ }^{1}$, Kye Sang Yoo ${ }^{2}$ and Joohan Kim ${ }^{1, *}$ \\ 1 Department of Mechanical Engineering, Graduate School, Seoul National University of Science \& Technology, \\ Seoul 01811, Korea; yoonsw@seoultech.ac.kr \\ 2 Department of Chemical \& Biomolecular Engineering, Graduate School, Seoul National University of Science \\ \& Technology, Seoul 01811, Korea; kyoo@seoultech.ac.kr \\ * Correspondence: joohankim@seoultech.ac.kr; Tel.: +82-2-970-6303
}

check for

updates

Citation: Yoon, S.; Yoo, K.S.; Kim, J. Effect of Pd Ions on the Generation of $\mathrm{Ag}$ and $\mathrm{Au}$ Heterogeneous

Nanoparticles Using Laser Ablation

in Liquid. Appl. Sci. 2021, 11, 1394.

https://doi.org/10.3390/app11041394

Academic Editor: Raed Abu-Reziq

Received: 8 January 2021

Accepted: 1 February 2021

Published: 4 February 202

Publisher's Note: MDPI stays neutral with regard to jurisdictional claims in published maps and institutional affiliations.

Copyright: (c) 2021 by the authors. Licensee MDPI, Basel, Switzerland. This article is an open access article distributed under the terms and conditions of the Creative Commons Attribution (CC BY) license (https:// creativecommons.org/licenses/by/ $4.0 /)$.
Featured Application: Heterogeneous nanoparticles can be applied in optoelectronics (as catalysts) and drug delivery systems. For bioengineering, living cells can be subjected to photothermal therapy using them (AgPd/AuPd). These heterosynthetic nanoparticles can enhance therapeutic efficiency.

Abstract: Heterogeneous Ag/Au nanoparticles combined with Pd ions were generated by irradiating $\mathrm{Ag} / \mathrm{Au}$ metal targets in a Pd solution with nanosecond and femtosecond lasers. AgPd and AuPd nanoparticles were generated by laser fragmentation and bonded. We numerically analyzed the hot spots with electromagnetic field enhancement of nanoparticles of different sizes separated by various distances. AgPd and AuPd nanoparticles differing in diameter were generated and showed different characteristics compared to typical core-shell heterogeneous nanoparticles. Pd ions played an important role in the generation of nanoparticles in liquid via laser ablation. The femtosecond laser produced both pure and heterogeneous nanoparticles of uniform size. The nanosecond laser produced pure nanoparticles with a relatively non-uniform size, which developed into spherical heterogeneous nanoparticles with a uniform (small) size in the presence of Pd ions. These nanoparticles could optimize applications such as photothermal therapy and catalysis.

Keywords: laser ablation in liquid; Pd ions; $\mathrm{Ag}$; $\mathrm{Au}$; heterogeneous nanoparticles

\section{Introduction}

Nanoparticles are applied in optoelectronics (as catalysts) and drug delivery systems because of their unique optical and chemical properties. Instead of nanoparticles composed of single elements; metal nanoparticle compositions are now being studied. Bimetallic particles may exhibit superior properties [1-5]. For nanoparticle-mediated photothermal treatment, Ag or Au nanoparticles have been preferred, but their material properties limit efficacy [6]. Optical properties and photothermal stability are improved if Ag or Au is combined with Pd [7]. Quintanilla et al. studied the light-to-heat conversion ability of AuPd nanoparticles [8]. In this study, it was found that Pd can act as an important element to increase the light-to-heat conversion efficiency depending on the bond type and amount. This means that the alloy containing the element Pd can be more effective in photothermal treatment. In addition, changes in the stability of nanoparticles with heat can be a problem in nanoparticle applications. Albrecht et al. confirmed that nano-octopods alloyed with Pd have improved stability compared to nanoparticles without Pd added at higher temperatures [9]. The AuPd heterometallic bond greatly improves catalytic and filtration performance. Heterogeneous bimetal nanoparticles may have the same structure as a metal alloy, metallic compound, or solid solution [10]. To date, most heterogeneous nanoparticles have been chemically synthesized, including via chemical reduction of an aqueous solution containing a surfactant. Metal shapes and sizes are determined by the 
surfactant and energy applied (ultrasonic waves or microwaves that induce nanoparticles in an apparatus that resembles a microwave oven) [11-14]. Compared to conventional heating, wave energy improves the energy transfer rate; direct heating enhances synthetic efficiency [15]. However, secondary liquid heating can be problematic, although test reproducibility is high.

The synthesis of metal nanoparticles in liquid via pulsed laser energy (a photochemical reaction) has been explored for decades [16-21]. It is possible to control the light wavelength and pulse width, and thus nanoparticle generation. Nanoparticles are produced at room temperature and are free of surfactant contamination. Nanoparticles several $\mathrm{nm}$ in size are easily generated. Complex nanoparticles of various shapes and sizes can be created from simple nanoparticles. The laser wavelength can range from the ultraviolet to the near-infrared, and the pulse width from femtoseconds to nanoseconds [22-24].

Nanoparticle generation via laser irradiation in liquid is classified as laser ablation in liquid (LAL) [25-29], laser fragmentation in liquid (LFL) [30], or laser melting in liquid (LML) [31,32] as shown in Figure 1. These methods can be further divided into top-down procedures that fracture a material when generating nanoparticles, and bottom-up approaches that incorporate an elemental material into new nanoparticles. Both mechanisms can be applied simultaneously. The principle underlying nanoparticle fabrication through ablation of a material using a focused high-energy laser is as follows. When the laser irradiates the material surface in liquid, nanoparticles are generated via near-field ablation, coulomb explosions, and photothermal ablation [33-36]. The nanoparticles thus created are then subject to the secondary effects of laser pulses, such as liquid shock waves. Typically, particles separated from the bulk material are fragmented by cavitation bubbles generated via LAL. In addition, the particles may repeatedly agglomerate to form homogeneous nanoparticles of various sizes $[23,24]$.

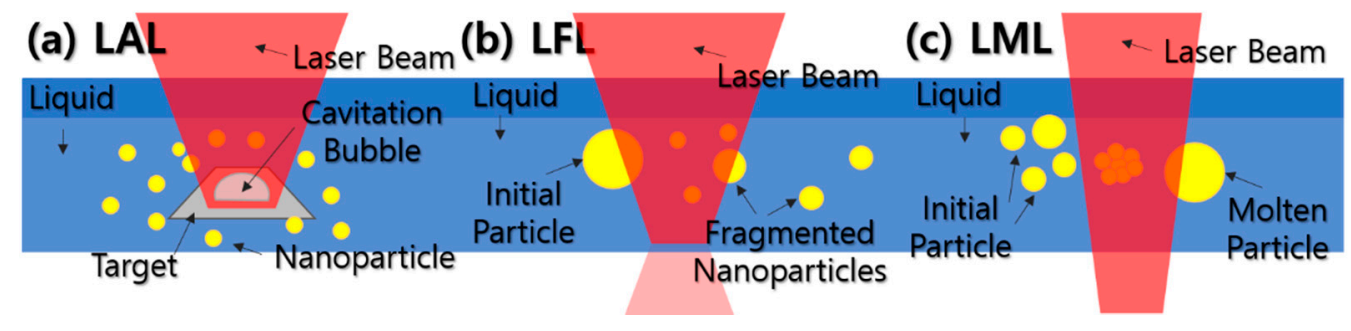

Figure 1. Schematics of (a) Laser ablation in liquid (LAL), (b) Laser fragmentation in liquid (LFL), and (c) Laser melting in liquid (LML).

Initially, nanoparticles are generated by irradiating a bulk metal. This is followed by a growth phase wherein the initial nanoparticles encounter laser pulses. Liquids can contain a variety of metal ions. When ablating a solid target, the ablation mechanism varies by the pulse width. When the laser is focused on a metal target, particle behavior is determined by the interaction time. At the same energy, interactions occurring over nanoseconds to microseconds trigger photothermal reactions; at shorter reaction times (femtoseconds to picoseconds), most reactions are photochemical, leading to nanoparticle growth or fragmentation [37-39]. Smaller and more homogeneous nanoparticles are generated at shorter pulse widths. However, for the bonding or growth of previously generated nanoparticles, nanosecond pulse widths that produce thermal effects may be important. In addition, plasma induced by laser ablation creates liquid shock waves and cavitation bubbles, which affect nanoparticle fragmentation and growth. Other metal ions in the liquid affect the sizes and shapes of the nanoparticles. The various mechanisms should ideally be independently examined, but this is very difficult given the extremely short time scales.

For bioengineering, living cells can be subjected to photothermal therapy using nanoparticles produced via LAL, LFL, and LML; heterosynthetic nanoparticles enhance 
therapeutic efficiency $[16,28,29,40]$. Silver (Ag) nanoparticles exhibit high laser absorption in the infrared range [40]. It is relatively easy to control the size of gold ( $\mathrm{Au}$ ) nanoparticles by adjusting the laser pulse width [41]. Palladium (Pd), which belongs to the platinum group, exhibits excellent electrical and thermal conductivity and ductility, and catalyzes both hydrogenation and dehydrogenation $[13,42]$. Heterogeneous nanoparticles may include combinations of these elements as alloys, intermetallic compounds, or core-shells, exhibiting new properties that complement those of single-element particles [3-5].

In a novel process, we synthesized heterogeneous ( $\mathrm{AgPd}, \mathrm{AuPd}$ ) nanoparticles with the characteristics of both elements via pulsed laser beam irradiation of $\mathrm{Ag}$ or $\mathrm{Au}$ in a solution of Pd ions. Femtosecond and nanosecond pulses were delivered, and solution metal ions affected nanoparticle fragmentation and growth. The process conditions were optimized, and the morphological characteristics size distribution, stability, and optical properties of the heterogeneous nanoparticles were analyzed.

\section{Modeling of Electric Field Concentration by Conducting Nanoparticles}

Electric field analysis is important when evaluating laser-induced nanoparticle fragmentation and growth. We modeled the behavior of multiple conducting nanoparticles exposed to an electric field. Variations in electric field strength caused by localized surface plasmon resonance (LSPR) coupling effect were analyzed according to the sizes of, particles and the distances between them. The simulation data aided interpretation of the experimental results. The electromagnetic field distribution caused by a laser in water was analyzed. The metallic nanoparticles were highly conductive and thus exhibited high current densities. Electromagnetic wave reduced its wave energy within nanoparticles and the electric field became concentrated around the conductors.

The distribution of the electric field by the incident laser beam can be approximated by a plane wave when comparing the sizes of the nanoparticles and the Gaussian beam. A Y-directed incident electric field can be described as follows:

$$
E=E_{0} \hat{y}
$$

Here, $E_{0}$ is the magnitude of the incident field and $\hat{y}$ is the unit vector in the $y$ direction. The electric field distribution $E$ can be expressed as a function of the electric potential $(\varphi)$ as:

$$
E=-\nabla \varphi
$$

To obtain this distribution, an analysis of the potential is required; this can be done using the following Laplace equation:

$$
\nabla^{2} \varphi=0
$$

For nanoparticles smaller than the wavelength of the laser, a quasi-static approximation is acceptable. When an electric field irradiates a nanoparticle, the electron clouds of atoms oscillate against the field because of Coulomb attraction by the atomic nucleus. The vibrating plasmon frequency is governed by the electron density clouds and a shape of nanoparticles. This plasmon frequency can be determined from the metal dielectric constant. We used the Lorenz-Drude model to approximate the dielectric function [43]:

$$
\varepsilon=\varepsilon_{0}+\sum_{j=1}^{M} \frac{G_{j} \omega_{p}^{2}}{\omega_{0 j}^{2}-\omega^{2}+i \Gamma_{j} \omega}, \sigma=0
$$

where $\varepsilon$ is the dielectric constant, $\varepsilon_{0}$ is the permittivity at infinite frequency, $\omega$ is the frequency of the incident electric field, $\omega_{p}$ is the plasmon frequency of the bulk metal, $G_{j}$ is the oscillator strength, and $\Gamma_{j}$ is the damping coefficient. To determine electric field distribution, we set boundary conditions for the governing equation above. The potential and electric displacement were continuous at the boundary of the particle [44]. In general, 
the electric field distribution of the above equation, which can be easily solved, is affected by the dipole field, including the particle dielectric function $\varepsilon$.

\section{Experimental}

First, Ag and Au targets (purity 99.99\%) were used to generate nanoparticles. LAL was done using $1 \mathrm{mM} \mathrm{Na}_{2} \mathrm{PdCl}_{4}$ solution; the control was $2 \mathrm{mM} \mathrm{NaCl}$ (both in deionized water). Precursor Pd is ionic in $\mathrm{Na}_{2} \mathrm{PdCl}_{4}$ solution and interacts with the laser to inhibit the growth of certain nanoparticles, but also to form AgPd and AuPd nanoparticles. The solid targets were fixed in the solution; laser ablation induced fragmentation and growth of heterogeneous nanoparticles. We used two laser beams. The femtosecond laser pulse width was $170 \mathrm{fs}$; after generation of a Ti:sapphire seed beam, the pulse energy was amplified. The nanosecond (fiber) laser pulse width was $5 \mathrm{~ns}$. Both beam wavelengths were in the near-infrared region (1064 and $1070 \mathrm{~nm}$, respectively). The pulse energy was 20 or $40 \mu \mathrm{J}$ for both lasers. The beams were delivered to metal targets fixed in the solution via a scanner with a focal length of $100 \mathrm{~mm}$. The laser spot size on the solid surface was $50 \mu \mathrm{m}$ and the laser fluence was approximately $1.0-2.1 \mathrm{~J} / \mathrm{cm}^{2}$.

The schematics of the process is shown in Figure 2. The process involved four steps (Figure 2a-d). A metal target was placed in the center of the container with the liquid, and pulsed laser beams were used to irradiate the target. The laser interacted with the target surface to cause ablation, and the plasma thus generated removed metal nanoparticles from the target. The detached nanoparticles were either further fragmented by laser pulses or reacted with elements in the plasma to form novel fused nanoparticles. As this sequence repeated, homogeneous and heterogeneous nanoparticles were produced.
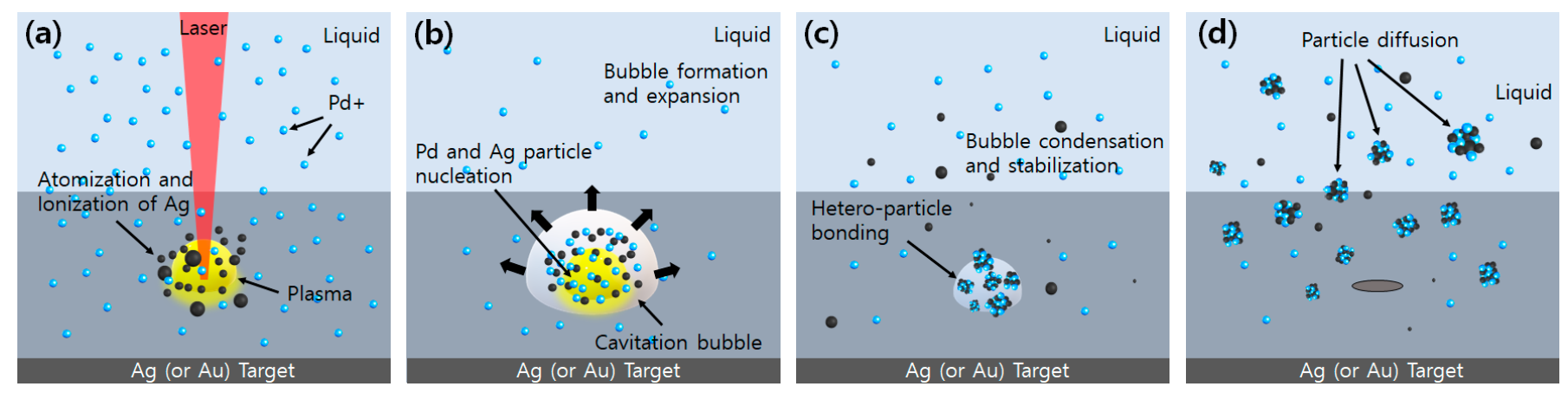

Figure 2. Schematic of process; (a) Pulsed laser beams irradiate the target and the laser interacted with the target surface to cause ablation. (b) The plasma and bubble with nanoparticles generate from the target. (c) Bubble condensation and heterogeneous nanoparticles occur. (d) The detached nanoparticles were either further fragmented by laser pulses or reacted with elements in the plasma to form novel fused nanoparticles.

The heterogeneous nanoparticles were collected and analyzed. Transmission electron microscope (TEM: JEM-2010; JEOL, Tokyo, Japan) images were acquired and the nanoparticle shapes were analyzed. The TEM operated at $200 \mathrm{kV}$; we also used an $\mathrm{LaB}_{6}$ electrode. About 500 800 nanoparticles on an image were analyzed in terms of the size distribution. Component analysis was performed using a TEM/energy-dispersive X-ray spectroscopy (TEM/EDS) instrument (INCA; Oxford Instruments, Abingdon, UK); the detector was $50 \mathrm{~mm}^{2}$ in area and the solid angle was $0.28 \mathrm{sr}$. We correlated the laser parameters with the levels of constituent elements in the nanoparticles.

\section{Experimental Results and Discussion \\ 4.1. Electric Field Concentration Simulation Analysis between Nanoparticles}

Simulation software (COMSOL Multiphysics; COMSOL Inc., Burlington, MA, USA) was used to analyze electromagnetic waves; the results are shown in Figure 3. On laser irradiation of metal nanoparticles, the conductive electrons oscillate because of SPR, thus 
concentrating the electric field near the nanoparticles. We analyzed the intensity changes of the electric field distributions around nanoparticles according to the distance between, and relative sizes of, the particles. We simulated cases 1-3 in Table 1. Circular conductive particles with mesh sizes of 3.7 and $133 \mathrm{~nm}$ were modeled. The meshes were free triangles.
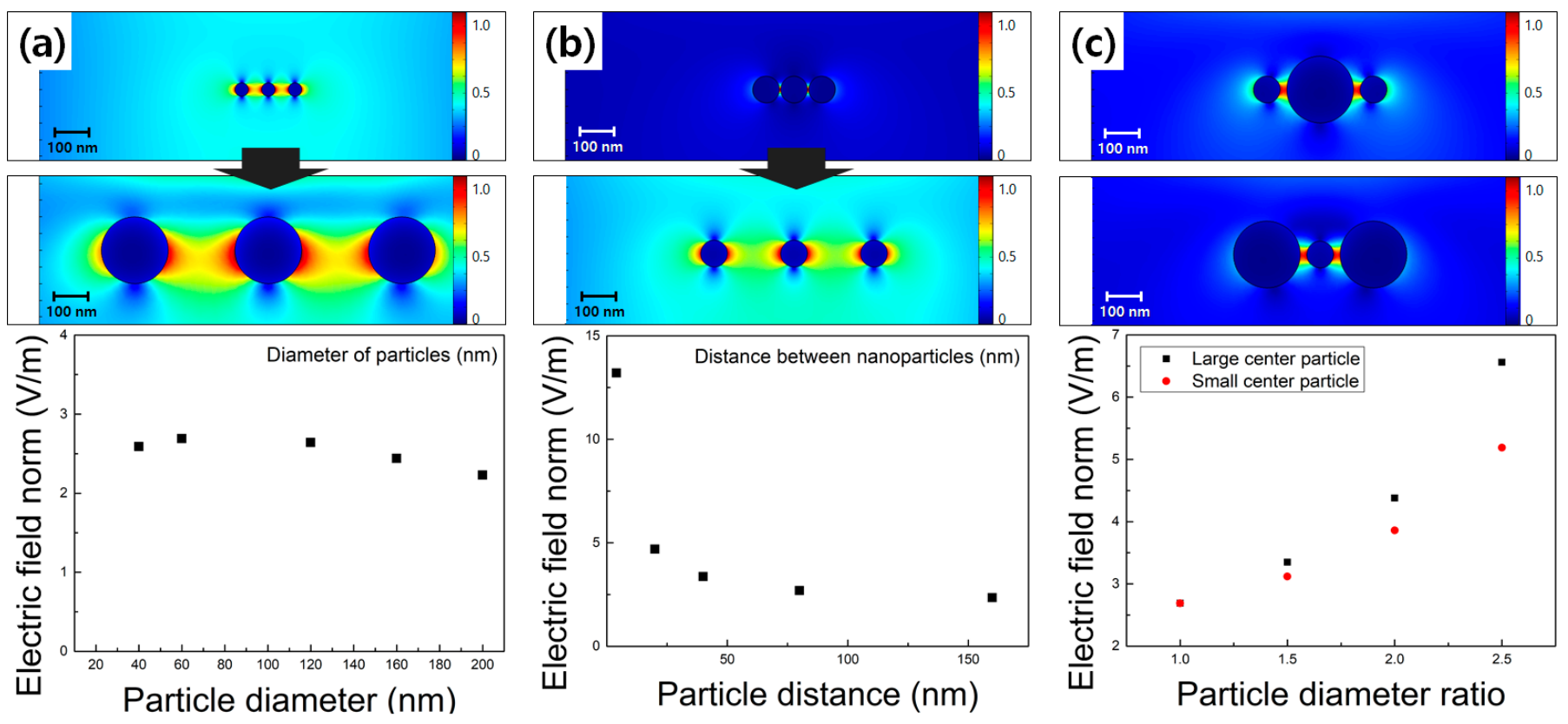

Figure 3. Simulated electric fields distribution and electric field vs. particle diameter. (a) Case 1, (b) Case 2, and (c) Case 3.

Table 1. Simulation parameters.

\begin{tabular}{ccc}
\hline Parameters & Values & Classification \\
\hline Particle Diameter $(\mathrm{nm})$ & $40 \sim 200$ & Case 1 \\
Distance between particles $(\mathrm{nm})$ & $4 \sim 160$ & Case 2 \\
The ratio of diameters between particles & $2 \mathrm{~d} \sim 4 \mathrm{~d}, 1 / 2 \mathrm{~d} \sim 1 / 4 \mathrm{~d}$ & Case 3 \\
\hline
\end{tabular}

Case 1 explored the effects of nanoparticle diameter (40-200 nm); as the diameter increased, the maximum electric field distributions around the particles were calculated as shown in Figure 3a. The characteristic particle length in this region was shorter than the wavelength, and the effect of hot spots with intense electromagnetic field enhancement according to absolute particle size was relatively constant [9,45-47]. The effect of the distance between nanoparticles on electric field concentration was analyzed in Case 2 (Figure $3 b$ ). The nanoparticle diameter used in the calculation was $40 \mathrm{~nm}$. As the particle spacing increased from 10 to $160 \mathrm{~nm}$, the maximum electric field intensity decreased dramatically. Therefore, the closer same-sized particles are to each other, the greater the electric field. Nanoparticles of different sizes were analyzed in Case 3 (Figure 3c). The size ratio of central and surrounding particles was 1:2.5. When the central particles were larger than the surrounding particles, the maximum electric field intensity was about $6-30 \%$ greater than in the opposite case. Thus, the simulations revealed the behaviors of differently sized nanoparticles as the electric field distribution varied. When particles were relatively close and small particles surrounded large particles, the hot spots with electromagnetic field enhancement were stronger than when large particles surrounded small particles. Thus, when nanoparticles of different sizes absorb laser energy, the electric field concentration is more efficient than when the particles are of the same size. This promotes nanoparticle generation and growth. If the pulse is relatively long, the electric field distribution acceler- 
ates the interactions between particles by instantaneously increasing the temperature of the particle surfaces, because the electric field becomes concentrated on the surfaces.

\subsection{TEM Images of Heterogeneous Nanoparticles}

We considered the following mechanism via which AgPd and AuPd may form alloys. When a laser beam irradiates a solid target, particles are generated by ablation and $\mathrm{Pd}$ ions reduced by electrons through laser irradiation are precipitated almost simultaneously. Both the particles and the precipitated Pd are affected by subsequent laser pulses, which concentrate the electric field around the nanoparticles, which thus become nano-sized alloys. If the pulse width is over nanoseconds, it the nanoparticles should reach thermal equilibrium. In such a case, the nanoparticles could grow due to diffusion. However, our pulse width was relatively short such as femtoseconds, so such nanoparticle growth was not possible.

Figure 4 shows TEM images of nanoparticles of various sizes and shapes created by varying the laser pulse, target material, and metal ions in solution. When there were no metal ions, most nanoparticles were spheres or polyhedrons (Figure $4 a, b$ ). Most particles were independent, but some were connected in chains, particularly Au particles. The Ag and Au nanoparticles were $5-30 \mathrm{~nm}$ in diameter. The shapes and sizes of particles may depend on the laser irradiation time, but we did not vary this parameter.
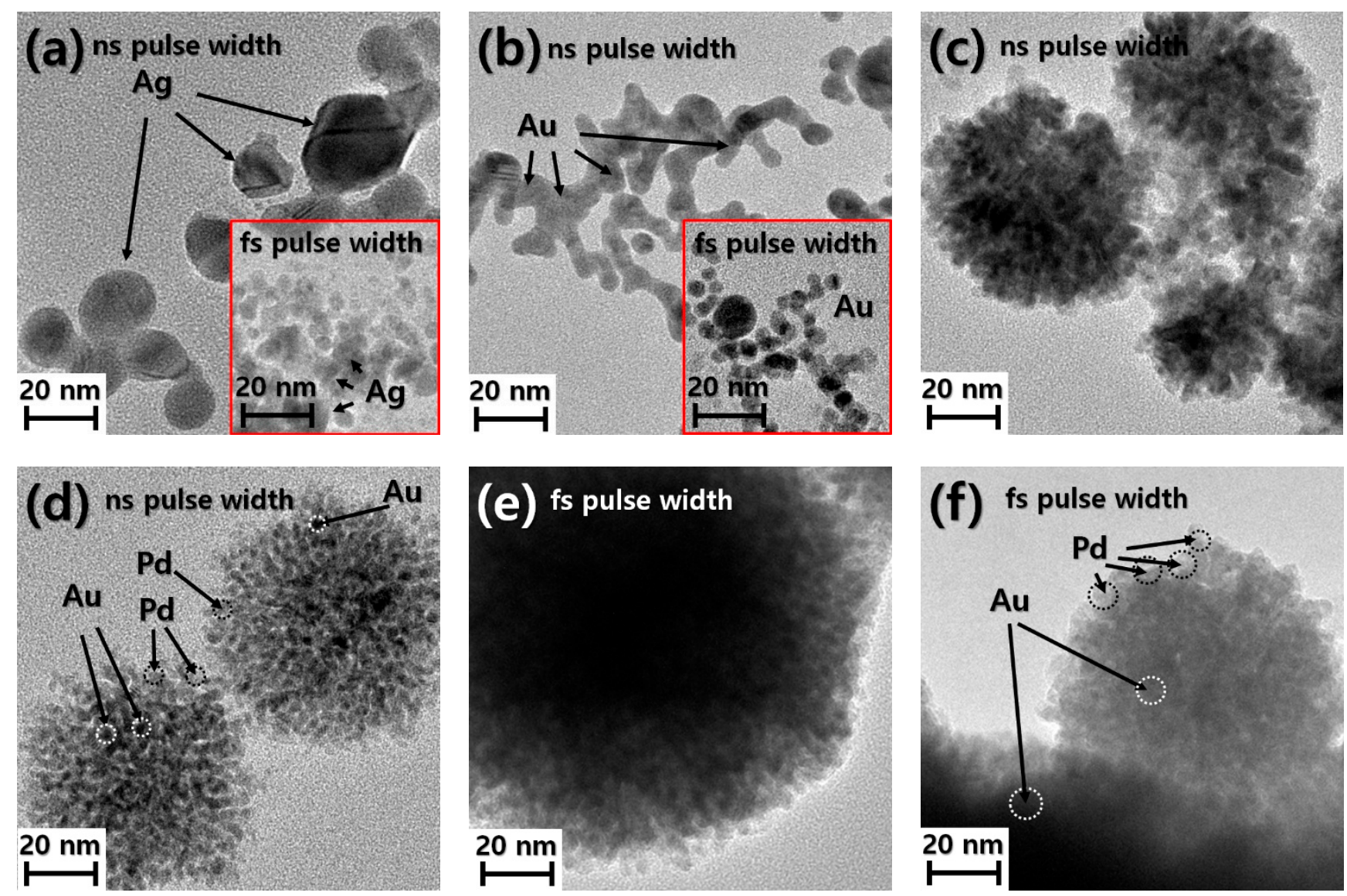

Figure 4. TEM images of synthesized nanoparticles. (a) Ag particles of nanosecond process and femtosecond process, (b) $\mathrm{Au}$ particles of nanosecond process and femtosecond process, (c) AgPd process particles of nanosecond process, (d) AuPd particles of nanosecond process, (e) AgPd particles of femtosecond, and (f) AuPd particles of femtosecond process.

The elements of heterogeneous nanoparticles can be distinguished in terms of brightness (light or dark). As the atomic numbers of Au and Pd differ greatly, these two elements can be distinguished. In general, an atom with a low atomic number exhibits high contrast. 
$\mathrm{Au}$ is darker than Pd. In the solution with Pd ions, the shapes and sizes of nanoparticles generated by the two lasers differed. In the presence of Pd ions, the shapes of $\mathrm{AgPd}$ and AuPd nanoparticles were random and colloidal; the clustered, heterogeneous nanoparticles had a diameter of 30-50 nm. This was particularly clear when nanosecond pulses were delivered (Figure 4c,d). AgPd and AuPd particles synthesized by the femtosecond laser also formed similarly shaped heterogeneous nanoparticle clusters, in which metal and Pd nanoparticles were homogeneously mixed (Figure 4e,f). However, femtosecond-generated heterogeneous nanoparticle clusters were about 1.5 to 2.5 times larger than nanosecondgenerated clusters (cf. Figure 4c-f). The effect of Pd ions on an Ag target can be understood by comparing Figure $4 \mathrm{a}$ to Figure $4 \mathrm{c}, \mathrm{e}$, and Figure $4 \mathrm{~b}$ to Figure $4 \mathrm{~d}$,f. When Pd ions were present in solution, the size of the nanoparticles was $3-5 \mathrm{~nm}$ in diameter. This means smaller nanoparticles were produced in existence of $\mathrm{Pd}$ ions. These ions enhance LAL fragmentation, as predicted by modeling (Figure 3c). The metal ions induce LSPR coupling effect around the nanoparticles, thereby affecting their size and contributing to the formation of colonies of heterogeneous nanoparticles.

Such nanoparticle alloys would be expected to behave differently from the coreshell nanoparticles prepared via conventional synthesis. For the latter nanoparticles, the elemental properties of the shell are stronger than those of the core when encountering waves delivered by a laser or microwave. However, the properties of both metals of an alloy will affect the responses to wave energy. In addition, the fact that the core and shell are formed from different metals limits nanoparticle size, but not the sizes of heterogeneous nanoparticles that are alloys. Even heterogeneous nanoparticles made of the same elements would be expected to differ in terms of their chemical and optical properties, depending on the bond type.

\subsection{Size Distribution of Heterogeneous Nanoparticles}

Figure 5 shows the particle size distributions of the various heterogeneous nanoparticles. Figure $5 \mathrm{a}, \mathrm{b}$ show the size distributions of $\mathrm{Ag}$ and Au nanoparticles generated using the nanosecond pulsed laser, and Figure $5 c, d$ the size distributions of nanoparticles generated using the femtosecond pulsed laser. Ag and Au nanoparticles without Pd ions created using the nanosecond and the femtosecond lasers had an average diameter of around 9.8 and $5.5 \mathrm{~nm}$, respectively. The particle size deviations were relatively large, at 9.0 and $1.5 \mathrm{~nm}$, respectively; thus, they were not uniform. In contrast, nanoparticles generated by femtosecond pulses had smaller diameters (3.6 and $3.7 \mathrm{~nm}$ for Ag and Au, respectively); they were also relatively uniform, with deviations of 0.7 and $0.8 \mathrm{~nm}$, respectively. When $\mathrm{Ag}$ and $\mathrm{Au}$ nanoparticles formed in the presence of Pd ions, most were 3-4 nm in size irrespective of the laser pulse width. Thus, the average particle sizes of 5.5 and $9.8 \mathrm{~nm}$ in the absence of Pd decreased 1.9-2.5-fold in the presence of Pd, and particle uniformity was greatly enhanced, consistent with the simulation results. When small and large nanoparticles are randomly mixed, fragmentation seems to increase because of hot spots with electromagnetic field enhancement. When using the femtosecond laser process, this is difficult to discern because the original particles generated are both uniform and small. The nanosecond laser reveals the electric field concentration effect much more clearly; the addition of appropriate metal ions rendered small nanoparticles uniform in size. 

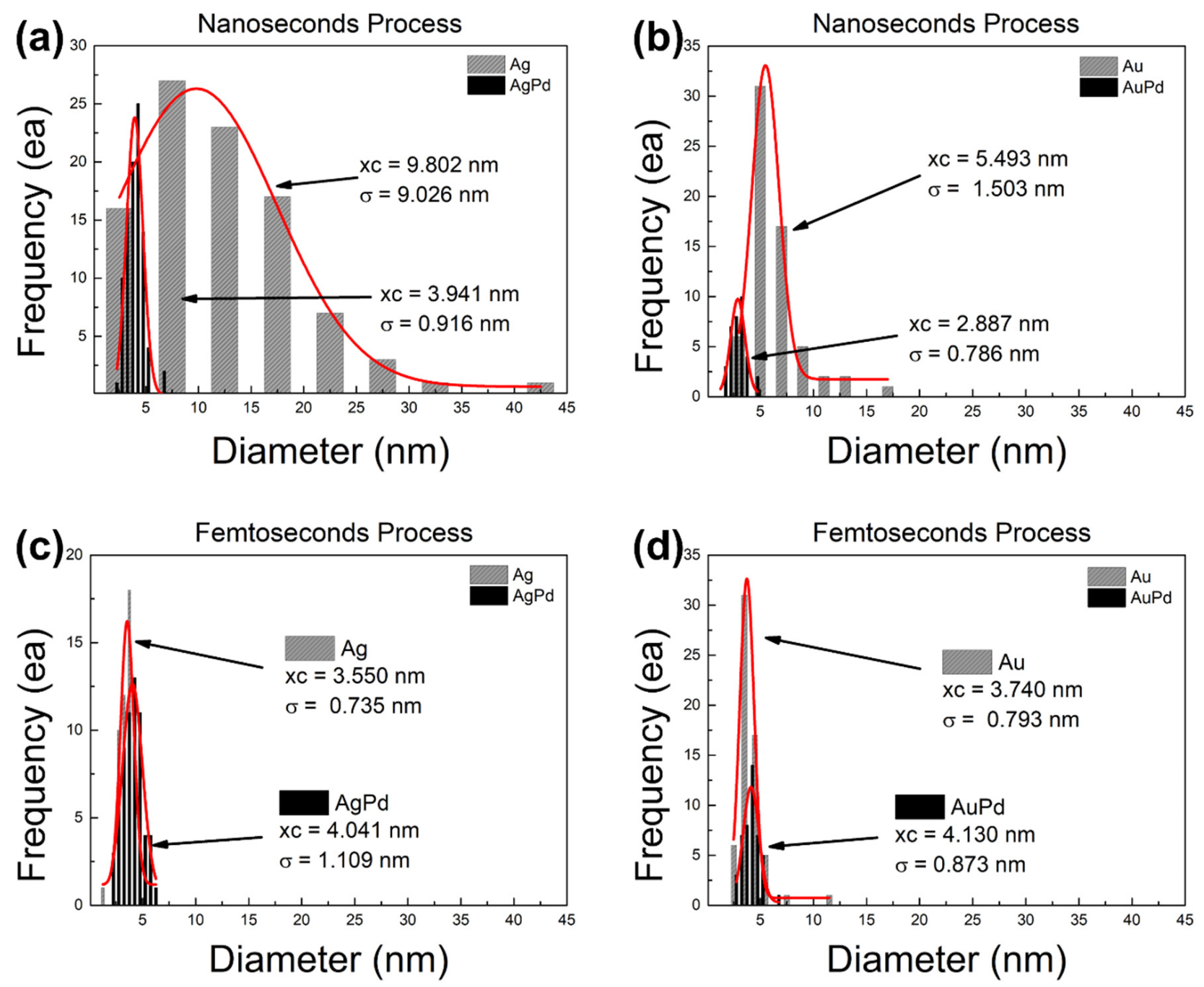

Figure 5. Size distributions of synthesized nanoparticles made by (a) nanoseconds laser pulses (Ag and AgPd), (b) nanoseconds laser pulses ( $\mathrm{Au}$ and $\mathrm{AuPd}$ ), (c) femtoseconds laser pulses (Ag and AgPd), and (d) femtoseconds laser pulses (Au and $\mathrm{AuPd})$.

\subsection{Composition of Nanoparticles Using TEM-EDS}

Figure 6 shows the compositions of the various nanoparticles according to the TEM/EDS elemental weight percentages of $\mathrm{Pd}$. After nanosecond pulsing, the weight ratio of $\mathrm{Ag}$ and $\mathrm{Pd}$ was about 1:1, compared to 1:2 for Au and Pd. As the molecular weight of Au is about twice that of Ag, both AgPd and AuPd nanoparticles were formed. The femtosecond pulse $\mathrm{Pd}$ weight percentages were significantly smaller than those of the nanosecond pulses; the femtosecond pulses affected more Pd ions. Femtosecond pulses would be expected to trigger photochemical reactions, and nanosecond pulses photothermal reactions. The rate at which $\mathrm{Pd}$ ions are photochemically converted into particles by photochemical reactions is faster than that for $\mathrm{Au}$ and $\mathrm{Ag}$, but photothermal conversion is slower. Accordingly, through femtosecond pulsing, relatively large numbers of Pd ions were involved in fragmentation and growth. 

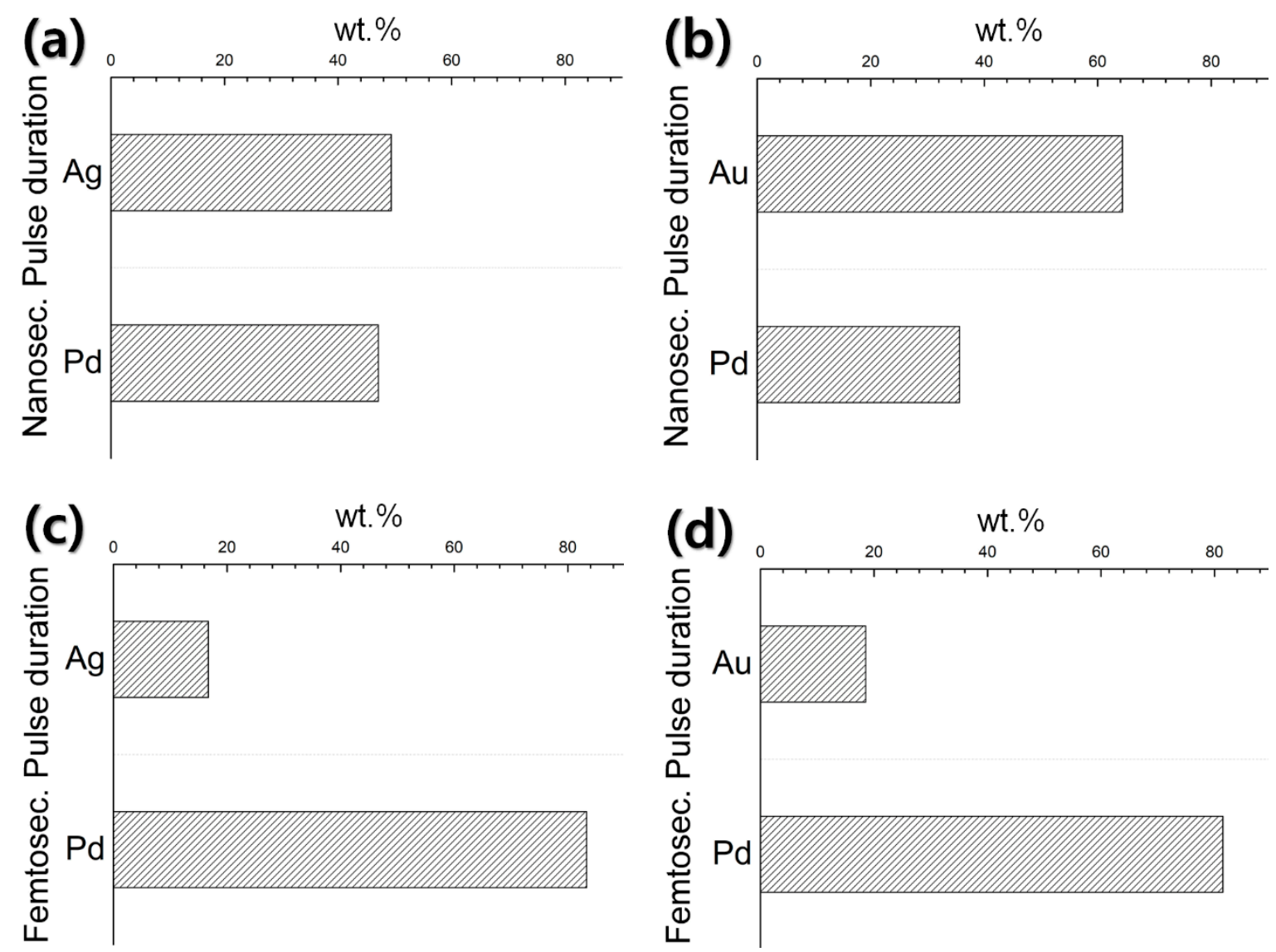

Figure 6. TEM-EDS results of nanoparticles (wt.\%) made by (a) nanoseconds laser pulses (AgPd), (b) nanoseconds laser pulses (AuPd), (c) femtoseconds laser pulses (AgPd), and (d) femtoseconds laser pulses (AuPd).

\section{Conclusions}

A study was carried out to synthesize AgPd and AuPd heterogeneous nanoparticles through laser irradiation on a metal target $(\mathrm{Ag} / \mathrm{Au})$ in a solution containing $\mathrm{Pd}$ ions. The resulting nanoparticles reach a stable shape by repeating their size reduction and growth under the influence of following laser pulses. In the synthesis of $\mathrm{Ag}$ or $\mathrm{Au}$ nanoparticles through LAL, it was confirmed that the presence of Pd ions affects the size, shape, and alloy ratio of the synthesized heterogeneous nanoparticles. An analysis of the electric field distribution for nanoparticles was performed to examine the effect on the electric field concentration between nanoparticles. The distance and relative size between nanoparticles influence the electric field distribution on and around the nanoparticle surface. In this case, it was expected that when relatively small nanoparticles such as Pd exist around the nanoparticles, the localized electric field concentration around the nanoparticles could be further strengthened. Considering these results, it seems that Pd ions can help fragmentation of nanoparticles and synthesis of heterogeneous nanoparticles. Through experiments, heterogeneous nanoparticles were synthesized using a nanosecond laser and a femtosecond laser. The morphology of the synthesized heterogeneous nanoparticles was confirmed through TEM, and a spherical alloy particle in which two elements were randomly distributed was observed. This type is expected to have different optical and chemical properties when compared to general core-shell heterogeneous nanoparticles. In the result of size distribution for the laser pulse width, pure nanoparticles were greatly affected by the laser pulse width. However, in the presence of Pd ions, the difference in the size of the particles with respect to the laser pulse width was not large, and heterogeneous nanoparticles of $3 \mathrm{~nm}$ to $4 \mathrm{~nm}$ were produced. Interestingly, Ag or Au particles with 
poor uniformity made by nanosecond lasers, in the presence of $\mathrm{Pd}$, were produced as heterogeneous nanoparticles of small uniform shape. This result is similar to the generation of heterogeneous nanoparticles by a femtosecond laser. Through TEM-EDS analysis, the relative synthesis ratio of $\mathrm{Pd}$ and $\mathrm{Ag} / \mathrm{Au}$ particles was quantitatively confirmed, and it was confirmed that the generation of Pd particles was accelerated in femtoseconds. In addition, it showed the effect of increasing the alloy ratio of $\mathrm{Pd}$ in the generation of heterogeneous nanoparticles when compared with the results at nanoseconds.

Author Contributions: Conceptualization and supervision, J.K.; experiment and analysis, S.Y.; evaluation, K.S.Y.; writing-review and editing S.Y., K.S.Y., and J.K. All authors have read and agreed to the published version of the manuscript.

Funding: This research was funded by Nation Research Foundation (NRF) of S. Korea, grant No.: NRF-2018R1D1A1B07041576.

Institutional Review Board Statement: Not applicable.

Informed Consent Statement: Not applicable.

Data Availability Statement: Data sharing is not applicable to this article.

Conflicts of Interest: The authors declare no conflict of interest.

\section{References}

1. Zhang, J.; Worley, J.; Dénommée, S.; Kingston, C.; Jakubek, Z.J.; Deslandes, Y.; Botton, G.A. Synthesis of metal alloy nanoparticles in solution by laser irradiation of a metal powder suspension. J. Phys. Chem. B 2003, 107, 6920-6923. [CrossRef]

2. Zhang, D.; Ma, Z.; Spasova, M.; Yelsukova, A.E.; Lu, S.; Farle, M.; Wiedwald, U.; Gökce, B. Formation Mechanism of LaserSynthesized Iron-Manganese Alloy Nanoparticles, Manganese Oxide Nanosheets and Nanofibers. Part. Part. Syst. Charact. 2017, 34, 1600225. [CrossRef]

3. Srinoi, P.; Chen, Y.T.; Vittur, V.; Marquez, M.D.; Lee, T.R. Bimetallic nanoparticles: Enhanced magnetic and optical properties for emerging biological applications. Appl. Sci. 2018, 8, 1106. [CrossRef]

4. Chaudhuri, R.G.; Paria, S. Core/Shell Nanoparticles: Classes, Properties, Synthesis Mechanisms, Characterization, and Applications. Chem. Rev. 2011, 112, 2373-2433. [CrossRef] [PubMed]

5. Zhong, C.J.; Maye, M.M. Core-Shell Assembled Nanoparticles as Catalysts. Adv. Mater. 2001, 13, 1507-1511. [CrossRef]

6. Zhu, J. Surface plasmon resonance from bimetallic interface in Au-Ag core-shell structure nanowires. Nanoscale Res. Lett. 2009, 4, 977-981. [CrossRef]

7. Langhammer, C.; Yuan, Z.; Zorić, A.I.; Kasemo, B. Plasmonic Properties of Supported Pt and Pd Nanostructures. Nano Lett. 2006, 6, 833-838. [CrossRef]

8. Quintanilla, M.; Kuttner, C.; Smith, J.D.; Seifert, A.; Skrabalak, S.E.; Liz-Marzán, L.M. Heat generation by branched Au/Pd nanocrystals: Influence of morphology and composition. Nanoscale 2019, 11, 19561-19570. [CrossRef]

9. Albrecht, W.; Bladt, E.; Vanrompay, H.; Smith, J.D.; Skrabalak, S.E.; Bals, S. Thermal Stability of Gold/Palladium Octopods Studied in Situ in 3D: Understanding Design Rules for Thermally Stable Metal Nanoparticles. ACS Nano 2019, 13, 6522-6530. [CrossRef]

10. Ferrando, R.; Jellinek, J.; Johnston, R.L. Nanoalloys: From theory to applications of alloy clusters and nanoparticles. Chem. Rev. 2008, 108, 845-910. [CrossRef]

11. Cheng, Y.; Schiffrin, D.J. Electrodeposition of metallic gold clusters at the water/1,2-dichloroethane interface. J. Chem. Soc. Faraday Trans. 1996, 92, 3865-3871. [CrossRef]

12. Platt, M.; Dryfe, R.A.W.; Roberts, E.P. Controlled deposition of nanoparticles at the liquid-liquid interface. Chem. Commun. 2002, 20, 2324-2325. [CrossRef]

13. Belloni, J.; Mostafavi, M.; Remita, H.; Marignier, J.L.; Delcourt, M.O. Radiation-induced synthesis of mono-and multi-metallic clusters and nanocolloids. New. J. Chem 1998, 22, 1239-1255. [CrossRef]

14. Gaddy, G.A.; Korchev, A.S.; McLain, J.L.; Slaten, B.L.; Steigerwalt, E.S.; Mills, G. Light-induced formation of silver particles and clusters in crosslinked PVA/PAA films. J. Phys. Chem. B 2004, 108, 14850-14857. [CrossRef]

15. Bradley, J.S.; Via, G.H.; Bonneviot, L.; Hill, E.W. Infrared and EXAFS Study of Compositional Effects in Nanoscale Colloidal Palladium-Copper Alloys. Chem. Mater. 1996, 8, 1895-1903. [CrossRef]

16. Zhang, D.; Gökce, B.; Barcikowski, S. Laser Synthesis and Processing of Colloids: Fundamentals and Applications. Chem. Rev. 2017, 117, 3990-4103. [CrossRef]

17. Menazea, A.A.; Elashmawi, I.; El-Kader, F.H.A.; Hakeem, N.A. Nanosecond Pulsed Laser Ablation in Liquids as New Route for Preparing Polyvinyl Carbazole/Silver Nanoparticles Composite: Spectroscopic and Thermal Studies. J. Inorg. Organomet. Polym. Mater. 2018, 28, 2564-2571. [CrossRef] 
18. Rodrigues, C.J.; Bobb, J.A.; John, M.G.; Fisenko, S.P.; El-Shall, M.S.; Tibbetts, K.M. Nucleation and growth of gold nanoparticles initiated by nanosecond and femtosecond laser irradiation of aqueous [ $\left.\mathrm{AuCl}_{4}\right]$. Phys. Chem. Chem. Phys. 2018, 20, 28465-28475. [CrossRef] [PubMed]

19. John, M.G.; Tibbetts, K.M. One-step femtosecond laser ablation synthesis of sub-3 nm gold nanoparticles stabilized by silica. Appl. Surf. Sci. 2019, 475, 1048-1057. [CrossRef]

20. Nishi, T.; Hayasaka, Y.; Nakamura, T.; Morikawa, T.; Sato, S. Core-shell like Au-Ir nanoparticles with spatially variant elec-tronic state of Au synthesized by femtosecond laser irradiation of solution. Appl. Surf. Sci. 2018, 457, 1044-1049. [CrossRef]

21. Amendola, V.; Meneghetti, M. Laser ablation synthesis in solution and size manipulation of noble metal nanoparticles. Phys. Chem. Chem. Phys. 2009, 11, 3805-3821. [CrossRef] [PubMed]

22. Heinz, M.; Srabionyan, V.V.; Avakyan, L.; Bugaev, A.L.; Skidanenko, A.V.; Kaptelinin, S.Y.; Ihlemann, J.; Meinertz, J.; Patzig, C.; Dubiel, M.; et al. Formation of bimetallic gold-silver nanoparticles in glass by UV laser irradiation. J. Alloys Compd. 2018, 767, 1253-1263. [CrossRef]

23. Zhang, D.; Choi, W.; Jakobi, J.; Kalus, M.-R.; Barcikowski, S.; Cho, S.-H.; Sugioka, K. Spontaneous Shape Alteration and Size Separation of Surfactant-Free Silver Particles Synthesized by Laser Ablation in Acetone during Long-Period Storage. Nanomaterials 2018, 8, 529. [CrossRef] [PubMed]

24. Zhang, D.; Choi, W.; Oshima, Y.; Wiedwald, U.; Cho, S.H.; Lin, H.P.; Sugioka, K. Magnetic Fe@ FeOx, Fe@ C and $\alpha$-Fe $2 \mathrm{O}_{3}$ single-crystal nanoblends synthesized by femtosecond laser ablation of fe in acetone. Nanomaterials 2018, 8, 631. [CrossRef]

25. Zhang, D.; Gökce, B.; Sommer, S.; Streubel, R.; Barcikowski, S. Debris-free rear-side picosecond laser ablation of thin ger-manium wafers in water with ethanol. Appl. Surf. Sci. 2016, 367, 222-230. [CrossRef]

26. Kruusing, A. Underwater and water-assisted laser processing: Part 1 -General features, steam cleaning and shock processing. Opt. Lasers. Eng. 2004, 41, 307-327. [CrossRef]

27. Kruusing, A. Underwater and water-assisted laser processing: Part 2-Etching, cutting and rarely used methods. Opt. Lasers Eng. 2004, 41, 329-352. [CrossRef]

28. Shih, C.Y.; Shugaev, M.V.; Wu, C.; Zhigilei, L.V. The effect of pulse duration on nanoparticle generation in pulsed laser ablation in liquids: Insights from large-scale atomistic simulations. Phys. Chem. Chem. Phys. 2020, 22, 7077-7099. [CrossRef]

29. Kanitz, A.; Kalus, M.R.; Gurevich, E.L.; Ostendorf, A.; Barcikowski, S.; Amans, D. Review on experimental and theoretical investigations of the early stage, femtoseconds to microseconds processes during laser ablation in liquid-phase for the synthesis of colloidal nanoparticles. Plasma. Sources Sci. Technol. 2019, 28, 103001. [CrossRef]

30. Lau, M.; Barcikowski, S. Quantification of mass-specific laser energy input converted into particle properties during pico-second pulsed laser fragmentation of zinc oxide and boron carbide in liquids. Appl. Surf. Sci. 2015, 348, 22-29. [CrossRef]

31. Rehbock, C.; Zwartscholten, J.; Barcikowski, S. Biocompatible Gold Submicrometer Spheres with Variable Surface Texture Fabricated by Pulsed Laser Melting in Liquid. Chem. Lett. 2014, 43, 1502-1504. [CrossRef]

32. Wang, H.; Pyatenko, A.; Kawaguchi, K.; Li, X.; Swiatkowska-Warkocka, Z.; Koshizaki, N. Selective Pulsed Heating for the Synthesis of Semiconductor and Metal Submicrometer Spheres. Angew. Chem. 2010, 122, 6505-6508. [CrossRef]

33. Werner, D.; Furube, A.; Okamoto, T.; Hashimoto, S. Femtosecond laser-induced size reduction of aqueous gold nanoparti-cles: In situ and pump- probe spectroscopy investigations revealing Coulomb explosion. J. Phys. Chem. C 2011, 115, 8503-8512. [CrossRef]

34. Kamat, P.V.; Flumiani, M.; Hartland, G.V. Picosecond Dynamics of Silver Nanoclusters. Photoejection of Electrons and Fragmentation. J. Phys. Chem. B 1998, 102, 3123-3128. [CrossRef]

35. Link, S.; Burda, C.; Mohamed, M.B.; Nikoobakht, B.; El-Sayed, M.A. Femtosecond transient-absorption dynamics of colloi-dal gold nanorods: Shape independence of the electron-phonon relaxation time. Phys. Rev. B 2000, 61, 6086. [CrossRef]

36. Besner, S.; Kabashin, A.V.; Meunier, M. Fragmentation of colloidal nanoparticles by femtosecond laser-induced supercon-tinuum generation. Appl. Phys. Lett. 2006, 89, 233122. [CrossRef]

37. Neuenschwander, B.; Jaeggi, B.; Schmid, M.; Hennig, G. Surface Structuring with Ultra-short Laser Pulses: Basics, Limitations and Needs for High Throughput. Phys. Procedia 2014, 56, 1047-1058. [CrossRef]

38. Kawasaki, M.; Masuda, K. Laser fragmentation of water-suspended gold flakes via spherical submicroparticles to fine nanoparticles. J. Phys. Chem. B 2005, 109, 9379-9388. [CrossRef] [PubMed]

39. Kim, H.-Y.; Yoon, S.; Choi, H.W.; Kim, J.; Farson, D.F.; Cho, S.-H. The Effect of Laser Pulse Widths on Laser-Ag Nanoparticle Interaction: Femto- to Nanosecond Lasers. Appl. Sci. 2018, 8, 112. [CrossRef]

40. El-Hussein, A.; Mfouo-Tynga, I.; Abdel-Harith, M.; Abrahamse, M.H.A.H. Comparative study between the photodynamic ability of gold and silver nanoparticles in mediating cell death in breast and lung cancer cell lines. J. Photochem. Photobiol. B Biol. 2015, 153, 67-75. [CrossRef]

41. Torrisi, L.; Restuccia, N. Laser-Generated Au Nanoparticles for Bio-Medical Applications. IRBM 2018, 39, 307-312. [CrossRef]

42. Semaltianos, N.G.; Chassagnon, R.; Moutarlier, V.; Blondeau-Patissier, V.; Assoul, M.; Monteil, G. Nanoparticles alloying in liquids: Laser-ablation-generated $\mathrm{Ag}$ or Pd nanoparticles and laser irradiation-induced AgPd nanoparticle alloying. Nanotechnology 2017, 28, 155703. [CrossRef] [PubMed]

43. Ali, A.; Naqvi, Q.A.; Baqir, M.A. Investigation of the plasmon resonance of core-shell nanoparticle in the near-infrared region. J. Electromagn. Waves Appl. 2019, 33, 2462-2475. [CrossRef] 
44. Kelly, K.L.; Coronado, E.; Zhao, L.L.; Schatz, G.C. The Optical Properties of Metal Nanoparticles: The Influence of Size, Shape, and Dielectric Environment. J. Phys. Chem. B 2003, 107, 668-677. [CrossRef]

45. Chen, S.; Meng, L.Y.; Shan, H.Y.; Li, J.F.; Qian, L.; Williams, C.T.; Tian, Z.Q. How to light special hot spots in multiparti-cle-film configurations. ACS Nano 2016, 10, 581-587. [CrossRef]

46. Schäfer, C.; Perera, P.N.; Laible, F.; Olynick, D.L.; Schwartzberg, A.M.; Weber-Bargioni, A.; Fleischer, M. Selectively ac-cessing the hotspots of optical nanoantennas by self-aligned dry laser ablation. Nanoscale 2020, 12, 19170-19177. [CrossRef]

47. Kuttner, C. Plasmonics in Sensing: From Colorimetry to SERS Analytics. In Plasmonics; Gric., T., Ed.; Books on Demand: Norderstedt, Germany, 2018; pp. 151-180. 